Session F

Convection and rotation 


\title{
Rotation of the solar convection zone from helioseismology
}

\author{
Jørgen Christensen-Dalsgaard ${ }^{1}$ \\ ${ }^{1}$ Institut for Fysik og Astronomi, Aarhus Universitet, DK-8000 Aarhus C, Denmark \\ email: jcd@phys.au.dk
}

\begin{abstract}
Helioseismology has provided very detailed inferences about rotation of the solar interior. Within the convection zone the rotation rate roughly shares the latitudinal variation seen in the surface differential rotation. The transition to the nearly uniformly rotating radiative interior takes place in a narrow tachocline, which is likely important to the operation of the solar magnetic cycle. The convection-zone rotation displays zonal flows, regions of slightly more rapid and slow rotation, extending over much of the depth of the convection zone and converging towards the equator as the solar cycle progresses. In addition, there is some evidence for a quasi-periodic variation in rotation, with a period of around $1.3 \mathrm{yr}$, at the equator near the bottom of the convection zone.
\end{abstract}

Keywords. Sun: oscillations, Sun: helioseismology, Sun: rotation, Sun: interior

\section{Introduction}

Convection and rotation are intimately linked in the solar convection zone. The convective timescales in the deeper parts of the convection zone are similar to the solar rotation period, giving rise to a strong influence of rotation on the convection dynamics. Also, transport of angular momentum by convection is likely important for the solar surface differential rotation and the interplay between convection and rotation presumably also causes other dynamical phenomena in the convection zone, such as the meridional circulation. Finally, interaction between convection, rotation and other flows are assumed to lead to the generation of the solar large-scale magnetic fields and their cyclic variation.

Before the advent of helioseismology, observation of solar rotation was limited to the motion of features across the solar disk, or measurements of the Doppler velocity of the solar surface plasma. This showed the variation in rotation with latitude, from a rotation period around 25 days at the equator to around 35 days near the pole, although the motion at high latitudes was difficult to follow. Also, differences in rotation rate between the surface plasma and magnetic features presumed to be anchored at different depths gave some indication of an increase of angular velocity with depth in the near-surface region (e.g., Wilcox \& Howard 1970; Foukal 1972).

Theoretical modelling through hydrodynamical simulations of the convection zone (Glatzmaier 1985; Gilman \& Miller 1986) was able to reproduce the surface variation and tended to show an angular velocity depending only on the distance from the rotation axis; such 'rotation on cylinders' was expected from simple hydrodynamical arguments, leading to the Taylor-Proudman theorem (Pedlosky 1987). Modelling of rotation of the deep solar interior was extremely uncertain. From observations of other stars it is assumed that the Sun was rotating substantially more rapidly in its early life, with a loss of angular momentum through the magnetized solar wind coupled to the convection zone; however, it was unclear to what extent transport of angular momentum in the radiative interior would lead to a spin-down of the solar core. 
The observational situation has been changed dramatically by helioseismology. Already early analyses by Deubner et al. (1979) of high-degree five-minute oscillations showed the effect of the advection of the modes by rotation, providing an independent indication of an increase of the velocity with depth. Gough \& Toomre (1983) provided a more solid theoretical basis for the extraction of such subsurface velocity signals or sound-speed fluctuations from helioseismic data. With observations over the last decade from the GONG network (Harvey et al. 1996) and the MDI instrument on the SOHO spacecraft (Scherrer et al. 1995) we now have a detailed picture of rotation in the solar interior and its variation over a full 11-year cycle. Interestingly, this does not conform with the early models of rotation on cylinders in the convection zone; as discussed by Brun et al. (these proceedings) models of the convection zone are still not fully able to account for the inferred rotation profile. A striking feature is the sharply localized change from the latitudinally differential rotation in the convection zone to the nearly solid-body rotation in the radiative interior. This takes place in a narrow region, the so-called tachocline (Spiegel \& Zahn 1992) near the base of the convection zone, of likely substantial importance to the generation of the solar magnetic cycle.

Here I give a brief overview of the helioseismic analyses that have led to these inferences of solar internal rotation and discuss some of the results. A more extensive review of solar rotation and helioseismic investigations of it was given by Thompson et al. (2003), while Christensen-Dalsgaard \& Thompson (2007) discussed the observed properties of the tachocline region in more detail. A recent detailed review of observation and modelling of the dynamics of the solar convection zone was provided by Miesch (2005).

\section{Helioseismic inferences of rotation}

As a background for interpreting the helioseismic results a short introduction to helioseismology is probably useful. Extensive treatments of stellar oscillations have been provided by Unno et al. (1989) and Gough (1993), while Christensen-Dalsgaard (2002) reviewed the techniques and results of helioseismology.

\subsection{Properties of solar oscillations}

Solar oscillations are believed to be excited stochastically by near-surface convection. They are observed at periods between roughly fifteen and three minutes, with largest amplitudes near periods of five minutes. The amplitudes per mode are minute: at most around $20 \mathrm{~cm} \mathrm{~s}^{-1}$ in radial velocity and below a few parts per million in intensity. Even so, it has been possible to determine the mode frequencies with extremely high accuracy, thus forming the basis for the helioseismic analyses.

A mode of solar oscillations depends on co-latitude $\theta$ and longitude $\phi$ as a spherical harmonic, characterized by the degree $l$ and the azimuthal order $m$, with $|m| \leqslant l$. In addition, the mode is characterized by its radial order $n$ which provides a measure of the number of nodes in the radial direction. The angular frequency $\omega_{n l m}$ in general depends on all three wave numbers.

The observed modes of solar oscillations are acoustic (or p) modes or, at relatively high degree, surface gravity (or f) modes. The p modes are trapped between the photosphere and an inner turning point at a distance $r_{\mathrm{t}}$ from the centre, determined by

$$
\frac{c\left(r_{\mathrm{t}}\right)}{r_{\mathrm{t}}}=\frac{\omega}{\sqrt{l(l+1)}},
$$

where $c$ is the adiabatic sound speed. Thus high-degree modes are trapped near the surface while low-degree modes penetrate to the solar core. For the f modes the displacement 


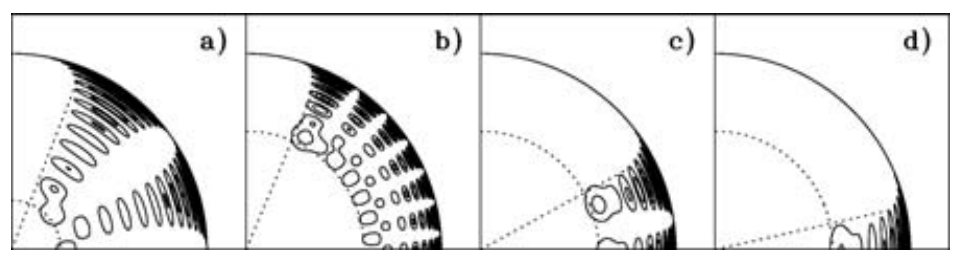

Figure 1. Contour plots of rotational kernels $K_{n l m}$ in a solar quadrant. The modes all have cyclic frequencies $\omega / 2 \pi$ near $2 \mathrm{mHz}$; the following pairs of $(l, m)$ are included: a) $(5,2) ; \mathrm{b})(20,8)$; c) $(20,17)$; and d) $(20,20)$. The dotted circles mark the locations of the lower radial turning point $r_{\mathrm{t}}$ (cf. eq. 2.1) and the dotted lines show the latitudinal turning points $\psi_{\mathrm{t}}$ (cf. eq. 2.2).

amplitude decreases exponentially with depth below the surface with an e-folding distance of approximately $R_{\odot} / l, R_{\odot}$ being the solar radius. The extent of the eigenfunctions in latitude is determined by the properties of the spherical harmonics; asymptotically, a mode is confined between latitudes of $\pm \psi_{\mathrm{t}}$, given by

$$
\cos \psi_{\mathrm{t}}=\frac{m}{l+1 / 2}
$$

thus modes with $m \simeq l$ are confined near the equator, whereas modes with $m / l \ll 1$ extend over all latitudes.

The Sun is rotating so slowly that the centrifugal force or other higher-order effects of rotation can be neglected. For the observed modes the dominant effect of rotation on the frequencies is simply the advection of the modes by rotation: the dependence of an oscillation on longitude and time $t$ can be written as $\cos \left(\omega_{n l m} t-m \phi\right)$, i.e., for $m \neq 0$ behaving as a wave running in the longitude direction; thus the frequencies of modes travelling in the direction of rotation are increased and frequencies of modes travelling in the direction opposite to rotation are decreased. It is plausible that the resulting frequencies are given by $\omega_{n l m} \simeq \omega_{n l 0}+m\langle\Omega\rangle$, where $\langle\Omega\rangle$ is a suitable average, determined by the properties of the mode, of the angular velocity $\Omega(r, \theta)$; as indicated, $\Omega$ is in general a function of the distance $r$ to the centre and $\theta$. A more careful analysis shows that the rotational splitting can be written as

$$
\delta \omega_{n l m} \equiv \omega_{n l m}-\omega_{n l 0}=m \int_{0}^{R_{\odot}} \int_{0}^{\pi} K_{n l m}(r, \theta) \Omega(r, \theta) r \mathrm{~d} r \mathrm{~d} \theta,
$$

where the kernels $K_{n l m}$ are determined by the structure of the Sun and the properties of the eigenfunctions. It is important to note that the kernels are symmetrical around the equator; thus the rotational splitting is only sensitive to the similarly symmetric component of $\Omega(r, \theta)$, and analysis of rotational splittings of global modes provides no information about the antisymmetric component of $\Omega$. Some examples of kernels are illustrated in Figure 1; it is evident that they largely follow the behaviour expected from the turning points in $r$ and $\theta$.

\subsection{Inverse analysis}

By observing a large selection of modes of such varying extent it is possible to make combinations of the rotational splittings that provide localized measures of $\Omega(r, \theta)$. Techniques for such inverse analyses were discussed by Schou et al. (1998). In many cases the inference of $\Omega\left(r_{0}, \theta_{0}\right)$ at some point $\left(r_{0}, \theta_{0}\right)$ is obtained as a linear combination of the data which, for example, could be taken on the form $m^{-1} \delta \omega_{n l m}$ :

$$
\Omega\left(r_{0}, \theta_{0}\right)=\sum_{n l m} c_{n l m}\left(r_{0}, \theta_{0}\right) m^{-1} \delta \omega_{n l m}=\int_{0}^{R_{\odot}} \int_{0}^{\pi} \mathcal{K}\left(r_{0}, \theta_{0}, r, \theta\right) \Omega(r, \theta) r \mathrm{~d} r \mathrm{~d} \theta
$$




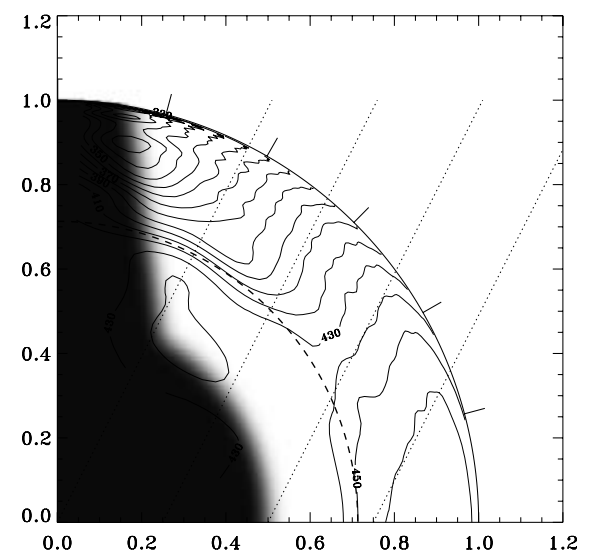

Figure 2. Inferred rotation rate $\Omega / 2 \pi$ in a quadrant of the Sun, obtained by means of inversion of 144 days of MDI data. The equator is at the horizontal axis and the pole is at the vertical axis, both axes being labelled by fractional radius. Some contours are labelled in $\mathrm{nHz}$, and, for clarity, selected contours are shown as bold. The dashed circle is at the base of the convection zone and the tick marks at the edge of the outer circle are at latitudes $15^{\circ}, 30^{\circ}, 45^{\circ}, 60^{\circ}$, and $75^{\circ}$. The shaded area indicates the region in the Sun where no reliable inference can be made with the present data. The slanted dotted lines are at an angle of $27^{\circ}$ with the rotation axis. (Adapted from Schou et al. 1998).

by using equation (2.3). Here the averaging kernel is given by

$$
\mathcal{K}\left(r_{0}, \theta_{0}, r, \theta\right)=\sum_{n l m} c_{n l m}\left(r_{0}, \theta_{0}\right) K_{n l m}(r, \theta) ;
$$

it is typically defined such as to have unit integral over $(r, \theta)$. Thus equation (2.4) defines the inferred angular velocity as an average which ideally is localized near $\left(r_{0}, \theta_{0}\right)$; the degree of localization is determined by the extent of the averaging kernel. Also, the error in $\Omega\left(r_{0}, \theta_{0}\right)$ can obviously be determined from equation (2.4) if the error properties of the data are known. How the inversion coefficients $c_{n l m}\left(r_{0}, \theta_{0}\right)$ are obtained depends on the properties of the specific inversion method; in some techniques they are found so as explicitly to localize the averaging kernel, whereas other techniques attempt to fit the inferred angular velocity to the observations in a least-squares sense (see Schou et al. (1998) for details).

\section{The solar internal rotation}

The inversion techniques discussed above have been extensively applied to observed solar oscillation frequencies. Already early results (Duvall et al. 1984) clearly demonstrated that the radiative interior rotated at roughly the surface rate, with little or no indication of a rapidly rotating core. With the determination of rotational splittings for different values of $m$ it became possible to obtain information about the variation of the latitudinally differential rotation with depth (Brown \& Morrow 1987; Christensen-Dalsgaard \& Schou 1988; Brown et al. 1989; Dziembowski et al. 1989), demonstrating the transition between differential rotation in the convection zone and near-constant rotation in the radiative interior. Extensive analyses by Schou et al. (1998) have showed a considerable degree of consistency between results of different inversion methods, although some sensitivity remains to the techniques used in the basic analysis to determine the rotational 


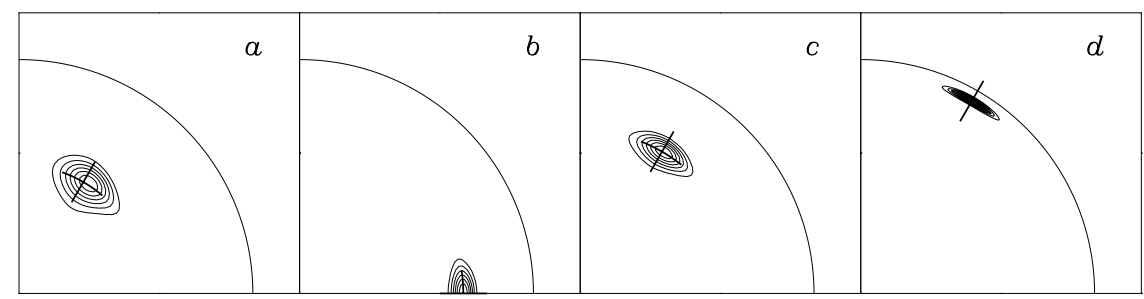

Figure 3. Averaging kernels for the inversion shown in Figure 2, targeted at the following radii and latitudes in the Sun: $\left(0.55 R_{\odot}, 60^{\circ}\right),\left(0.7 R_{\odot}, 0^{\circ}\right),\left(0.7 R_{\odot}, 60^{\circ}\right)$, and $\left(0.95 R_{\odot}, 60^{\circ}\right)$. The corresponding locations are indicated with crosses. (Adapted from Schou et al. 1998).

splittings from the observations (Schou et al. 2002), particularly at high latitudes. Using also data on low-degree modes from unresolved observations the absence of a rapidly rotating core has been confirmed, although the inferences are still uncertain in the inner around $20 \%$ of the solar radius (e.g., Chaplin et al. 1999). This confirms that an efficient mechanism must have linked the radiative interior to the loss of angular momentum from the convection zone; the nature of this mechanism is still debated, however.

\subsection{The bulk of the convection zone}

Here I concentrate on rotation within and just below the convection zone. Figure 2 shows an inferred rotation profile in the outer parts of the Sun. Some averaging kernels for this inversion are illustrated in Figure 3; evidently the combinations of the observed splittings are able to determine the angular velocity with fairly high resolution, particularly in the radial direction near the surface. In the bulk of the convection zone the latitudinal variation clearly roughly follows the variation at the surface, the helioseismically determined values close to the surface being essentially consistent with direct Doppler measurements of the surface rotation (e.g., Beck 2000). It is obvious that the rotation is not constant on cylinders, as simple models predicted. As noted by Gilman \& Howe (2003) it rather appears that in much of the convection zone rotation is constant on lines inclined at $27^{\circ}$ to the rotation axis; the physical reason for this property is not understood.

Very near the surface the contours clearly indicate that the rotation rate decreases outwards, at low and intermediate latitude. This corresponds to the behaviour inferred from surface markers presumed to be anchored at different depths and is reminiscent of the early results of Deubner et al. (1979). In a more careful analysis based on $\mathrm{f}$ modes Corbard \& Thompson (2002) determined the gradient of the angular velocity in this near-surface region, as shown in Figure 4. Interestingly, $\mathrm{d} \ln \Omega / \mathrm{d} \ln r \simeq-1$ at latitudes below around $40^{\circ}$; this is not consistent with the, perhaps natural, assumption of constant angular momentum maintained in this region by convective motions, which would instead correspond to $\mathrm{d} \ln \Omega / \mathrm{d} \ln r=-2$.

\subsection{Properties of the tachocline}

As already mentioned, the properties of the tachocline are of very considerable interest. Particularly important are the location and thickness of the transition, first addressed in detail by Kosovichev (1996). Determination of these properties must obviously take into account the resolution of the inversion, as characterized by the averaging kernels (cf. Figure 3). The effect on the inversion is illustrated in Figure 5a, on the basis of inversion of artificial data computed for the rotation profile shown by the heavy curves. The corresponding inversion results, indicated by thin curves and shaded areas, clearly show a transition that is more gradual than for the 'true' underlying rotation rate, as a result of the smearing by the averaging kernel. The result of the corresponding 


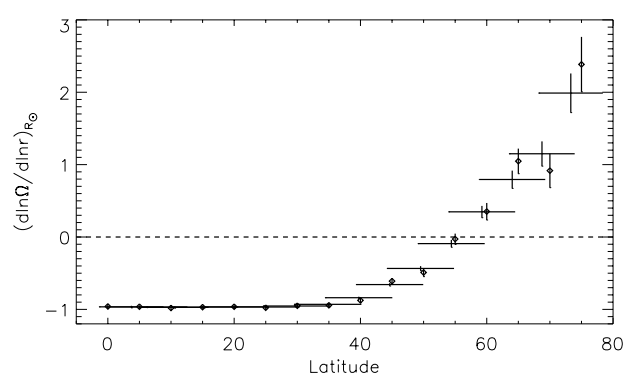

Figure 4. Logarithmic gradient of the angular velocity obtained from linear fits in the outer two per cent of the solar radius to the results of inversion of f-mode splittings. (Adapted from Corbard \& Thompson 2002).

inversion of solar data (Charbonneau et al. 1999), shown in Figure 5b, in fact indicates a width that roughly corresponds to the width assumed for the artificial data. Further information about the transition can be obtained, as discussed by Charbonneau et al., by arranging the linear combination of the data in equation (2.4) such that the corresponding combination $\mathcal{K}$ of the kernels provides an average of the radial gradient of $\Omega$. The result (Figure 5c) clearly shows the localized gradient in the tachocline, furthermore hinting that the transition takes place closer to the surface at latitude $60^{\circ}$ than at the equator.

From tests on artificial data, and from parametrized fits, it is to some extent possible to correct for the finite resolution (e.g., Charbonneau et al. 1999; Corbard et al. 1999), and hence obtain a measure of the actual width. For example, Basu \& Antia (2003) determined the location $r_{\mathrm{c}}$ of the midpoint of the transition and its width $w \dagger$ as $r_{\mathrm{c}}=$ $0.692 R_{\odot}, w=0.033 R_{\odot}$ at the equator and $r_{\mathrm{c}}=0.710 R_{\odot}, w=0.076 R_{\odot}$ at latitude $60^{\circ}$. Thus they confirmed the prolate nature of the transition and furthermore found that it is broader at high latitude than at the equator. For comparison, the radius at the base of the convection zone, assuming spherical symmetry, has been determined from helioseismology as $0.713 R_{\odot}$ (e.g., Christensen-Dalsgaard et al. 1991); thus, under this assumption, most of the transition takes place below the convection zone at the equator, whereas at higher latitude there is considerable overlap between the tachocline and the convection zone.

\section{Variations with time}

Doppler observations of the solar surface rotation have shown variations with time in the so-called torsional oscillations (Howard \& LaBonte 1980), bands of slightly faster and slower rotation that converge towards the equator as the solar cycle progresses. The availability of detailed helioseismic observations extending over a sunspot cycle now allows investigations of variations in solar structure, rotation and other types of dynamical phenomena beneath the solar surface. Particularly detailed investigations have been made of changes in rotation; the principle is to analyse the data in segments of typically $2-3$ months, and consider the residual from the time-averaged rotation rate, as a function of $r$ and $\theta$. A recent overview of the results was provided by Howe (2006).

$\dagger$ defined such that $84 \%$ of the transition takes place between $r_{\mathrm{c}}-w$ and $r_{\mathrm{c}}+w$ 


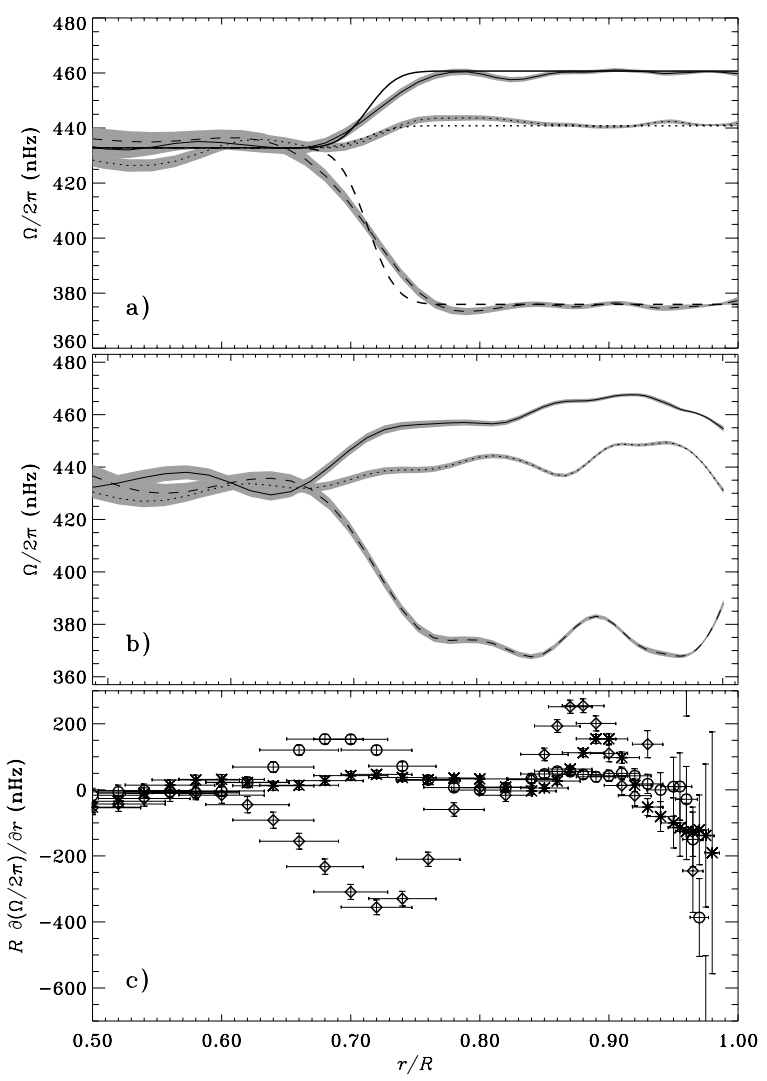

Figure 5. (a) Inversion of synthetic dataset for artificial rotation profile, based on artificial data with properties corresponding to an observed dataset. The thick curves show the underlying input rotation profile, in the form of radial cuts at latitudes of $60^{\circ}$ (dashed curve), $30^{\circ}$ (dotted curve), and $0^{\circ}$ (equator; solid curve). The thin curves show a typical inversion solution, with the $1 \sigma$ range, determined from the errors in the observed mode set, indicated by the shaded areas. (b) Corresponding inversion result for the solar data. (c) Results of inversion for the radial gradient of the solar rotation rate at latitude $0^{\circ}$ (circles), $30^{\circ}$ (asterisks) and $60^{\circ}$ (diamonds); here the vertical error bars indicate $1 \sigma$ errors, whereas the horizontal bars indicate the resolution of the inversion as determined by the extent in radius of the averaging kernels. (Adapted from Charbonneau et al. 1999).

\subsection{Tachocline oscillations?}

Perhaps the most surprising result of such analyses was the detection of what appeared to be an oscillation with a period of $1.3 \mathrm{yr}$ in the equatorial rotation rate near the base of the convection zone (Howe et al. 2000a). As shown in Figure 6, this oscillation was present, with largely consistent behaviour, in analyses of two different datasets and using two different inversion methods, between late 1995 and 2000, while the variation has been more erratic since then. The early period also appeared to show an oscillation with a similar period, but the opposite phase, at somewhat greater depth at the equator. Given the subtle nature of the signal, it is important to check whether it could be an artefact of, for example, variations in the selection of modes included in the analysis; this appears not to be the case (Toomre et al. 2003). However, Basu \& Antia (2001), while apparently detecting a similar variation, questioned its statistical significance. It is evident that the 


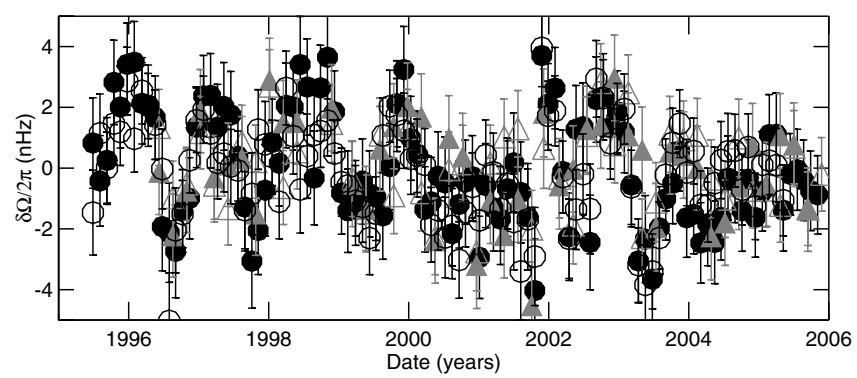

Figure 6. Residuals from average rotation rate at $r=0.72 R_{\odot}$ at the equator. Triangles and circles are based on MDI and GONG data, respectively, and open and closed symbols correspond to two different inversion techniques. (Adapted from Howe et al. 2007).

erratic nature of the variation may cast doubt on its physical reality; on the other hand, it is not implausible that such behaviour, of possibly magnetic origin, could be linked to specific phases in the solar cycle. Thus continued observations during the rising phase of the next cycle will be of great interest.

\subsection{Zonal flows}

Helioseismic investigations have showed that, far from being a superficial phenomenon, the torsional oscillations seen on the solar surface (Howard \& LaBonte 1980) extend quite deeply. From inversion of f modes Kosovichev \& Schou (1997) found that the regions of slightly faster and slower rotation extended a few per cent of the solar radius beneath the solar surface. Schou (1999) considered f-mode data covering more than two years and found that these subsurface zonal flows shared the propagation towards the equator seen on the surface, closely linked to the 'butterfly diagram' of the sunspots. More extensive data, on both p and f modes, were considered by Antia \& Basu (2000) and Howe et al. (2000b), who confirmed the equator-ward propagation of the zonal flows at low and intermediate latitude, the flow extending through up to one third of the convectionzone depth. At high latitude, on the other hand, flows of somewhat higher amplitude propagated towards the pole. Even deeper penetration, particularly at high latitude, was inferred by Vorontsov et al. (2002), using a non-linear inversion technique. They also found that the propagation could be fitted as having an 11-yr period and determined the amplitude and phase of this variation.

As an example of these results, Figure 7 compares flows from Doppler observations at the solar surface with helioseismically inferred flows at a depth of $0.01 R_{\odot}$. The two patterns are evidently very similar. The variation with depth is illustrated in Figure 8; this confirms that the flows can be followed through most of the convection zone. Also, strikingly, the flow pattern appears to propagate towards the solar surface as time progresses; this is difficult to reconcile with a model proposed by Spruit (2003) according to which the flow originates from thermal effects at the solar surface.

The origin of these zonal flows has been discussed in the context of mean-field dynamo models (e.g., Covas et al. 2000; Rempel 2006a,b). Rempel found that the pole-ward propagating branch could be explained by the feed-back of the Lorentz force on differential rotation in such models. He also showed that a similar mechanical model for the equator-ward propagating low-latitude branch would be strongly affected by the TaylorProudman theorem, leading to a variation with depth and latitude that is inconsistent with observations; thus he concluded that thermal effects must be involved. 

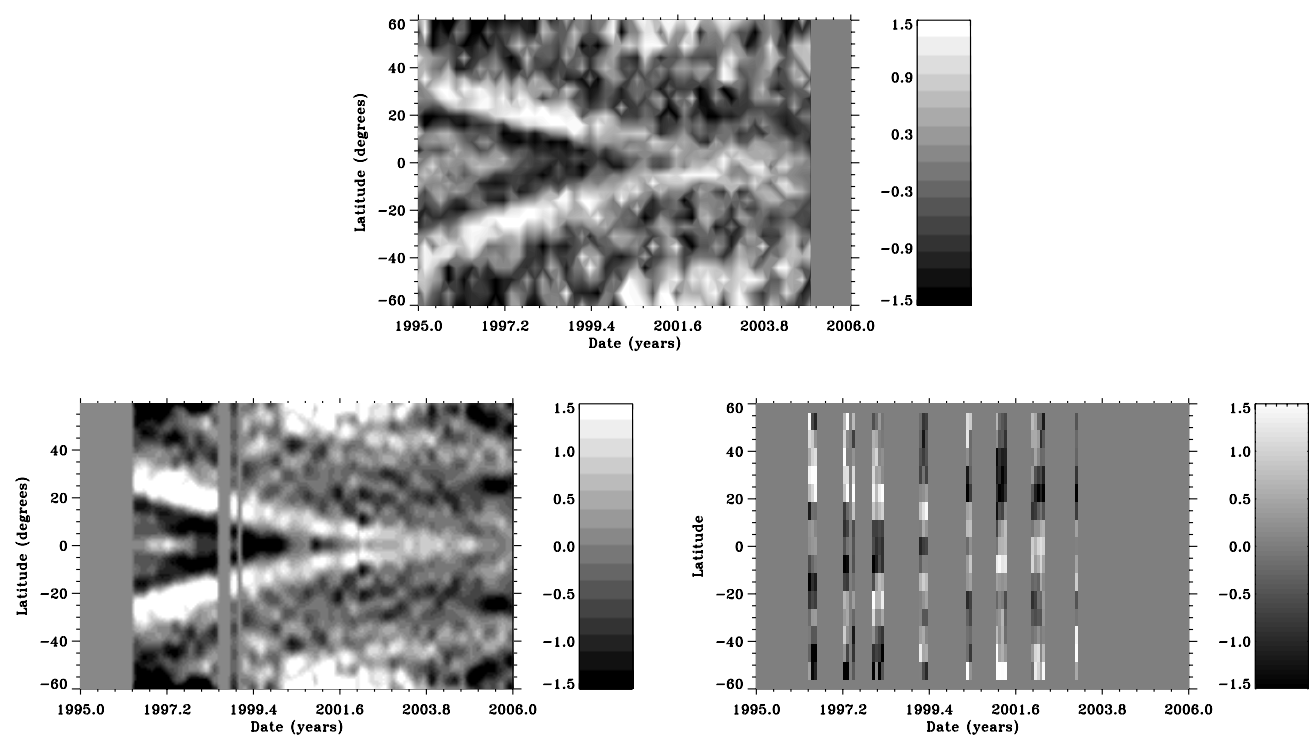

Figure 7. Zonal flow patterns, obtained as residuals from time-averaged rotation rates. The top panel shows the 'torsional oscillations' obtained from Mount Wilson surface Doppler observations. The two lower panels are from inverse analyses targeted at $r=0.99 R_{\odot}$; in the left panel global inversion of MDI data was used, whereas the right panel is based on a local helioseismic analysis with the ring-diagram technique (see Section 5), during time periods where high-resolution MDI data were available. (Adapted from Howe et al. 2006a).

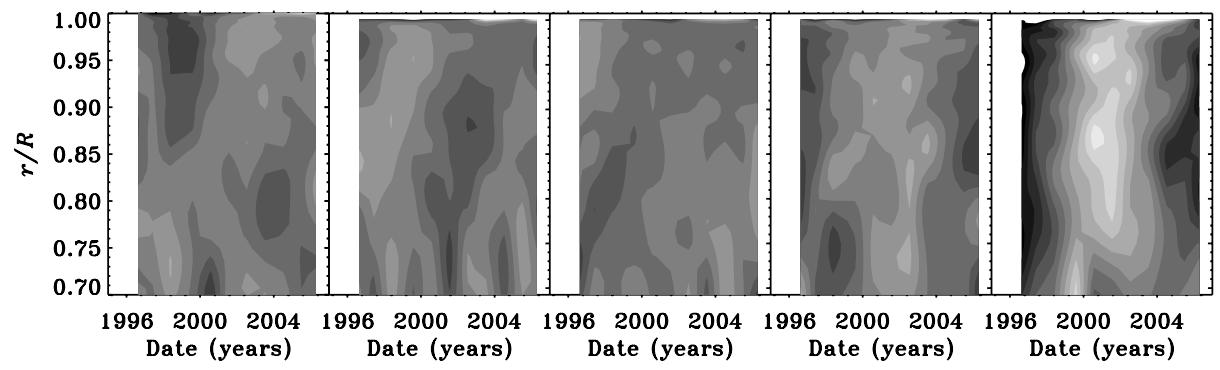

Figure 8. Rotation residuals as a function of time and fractional distance from the centre, from inversions of MDI data, at latitudes (left to right) $0^{\circ}, 15^{\circ}, 30^{\circ}, 45^{\circ}$ and $60^{\circ}$. The data have been averaged over periods of 1 yr. Darker regions correspond to rotation slower than average, and lighter regions to faster rotation. (Adapted from Howe et al. 2006b)

\section{Local helioseismology}

I have so far considered global helioseismology, based on frequencies of global modes of solar oscillation. This is restricted to consider only rotationally symmetric aspects of the solar interior structure and dynamics, such as rotation; furthermore, as noted in Section 2.1, the analysis is sensitive only to aspects that are symmetrical around the equator. These restrictions can be avoided through the use of local helioseismology where the data are analysed instead in terms of wave propagation in a smaller part of the solar surface. An extensive review of the techniques and results of such analyses was given by Gizon \& Birch (2005). 
Detailed investigations of sub-surface flows have been made with the ring-diagram technique, following the early analysis of Gough \& Toomre (1983). The oscillations are analysed in smaller patches on the solar surface, to produce local power spectra in terms of the horizontal wave vector $\mathbf{k}_{\mathrm{h}}$ (Hill 1988). By considering a large number of such patches distributed over the solar disk one can build up a picture of the subsurface structure and flows as a function of position on the Sun. A detailed investigation of this nature was carried out by Haber et al. (2002); they determined the longitudinally averaged rotational flow, also identifying the zonal flows. The results were similar to those of the global analyses, but with clear differences between the northern and southern hemispheres. This is also illustrated in Figure 7, where the lower right panel shows results obtained with a ring-diagram analysis. Similar results were obtained by Zhao \& Kosovichev (2004) using the time-distance technique, where a correlation analysis is used to infer the travel time of waves between different points on the solar surface.

Local helioseismology has also revealed other kinds of large-scale flows beneath the solar surface. An important example is the meridional circulation, which is also seen on the solar surface in Doppler-velocity observations (e.g., Hathaway 1996), such that the flow is generally in the pole-ward direction. Using time-distance analysis Giles et al. (1997) found a similar flow in the upper parts of the convection zone. This was analysed in more detail by Haber et al. (2002) and Zhao \& Kosovichev (2004) using ring-diagram and time-distance analyses, respectively. On smaller scales local analyses have revealed convective flows on supergranular scales, as well as complex flows associated with active regions (see also Kosovichev, these proceedings).

\section{Open questions}

While major progress has obviously been made on our knowledge about rotation in the solar interior, much remains to be done. The determination of the rotation of the inner solar core is still highly uncertain. Unfortunately, substantial improvement in the low-degree p-mode splittings, required to determine the core rotation, will require much more extensive observations than now available; observation of g modes, which are more sensitive to the properties of the solar core, remains elusive despite recent encouraging progress (García et al. 2006). Better observational constraints are also required on the properties of the tachocline region; this includes details of solar structure such as the depth of the convective envelope and the detailed properties of the transition to the radiative region, as well as a better determination of the location and thickness of the transition in rotation. Also, it is obviously important to test the reality of the $1.3 \mathrm{yr}$ oscillation in rotation in the tachocline region and investigate its diagnostic potential.

From a theoretical point of view, the evolution of rotation in the radiative interior of the Sun is still poorly understood, although there are models that are able to reproduce the present near-constant rotation through, for example, the effect of a dynamo-generated magnetic field (Eggenberger et al. 2005) or transport by gravity waves (Charbonnel \& Talon 2005). This issue is obviously closely related to the establishment of the tachocline, where magnetic fields have also been invoked as the most likely mechanism (e.g., Gough \& McIntyre 1998). Progress towards understanding the mean rotation profile within the convection zone has been made in terms of mean-field models which involve thermal effects in the tachocline region (Rempel 2005). Evidently, the preferred route to understand the dynamics of the convection zone would be through realistic three-dimensional simulations. Brun \& Toomre (2002) showed that increased numerical resolution in such simulations improved the agreement with the observed profile, and the simulations have confirmed that including entropy variations in the tachocline region can act to bring the 
results into better agreement with the helioseismic inferences (Miesch et al. 2006, see also Brun et al. these proceedings). Interestingly, detailed modelling of the outer $5 \%$ of the solar radius (DeRosa et al. 2002) has reproduced a near-surface decrease in the angular velocity, such as observed in the shear layer (cf. Figure 4). A definite model of the zonal flows is still lacking; one may hope that such flows will eventually emerge as natural features of the hydrodynamical simulations.

\section{Acknowledgements}

I am very grateful to T. Corbard and R. Howe for help with figures in this review.

\section{References}

Antia, H. M. \& Basu, S. 2000, ApJ 541, 442

Basu, S. \& Antia, H. M. 2001, MNRAS 324, 498

Basu, S. \& Antia, H. M. 2003, ApJ 585, 553

Beck, J. G. 2000, Solar Phys. 191, 47

Brown, T. M. \& Morrow, C. A. 1987, ApJ 314, L21

Brown, T. M., Christensen-Dalsgaard, J., Dziembowski, W. A., Goode, P., Gough, D. O. \& Morrow, C. A. 1989, ApJ 343, 526

Brun, A. S. \& Toomre, J. 2002, ApJ 570, 865

Chaplin, W. J., Christensen-Dalsgaard, J., Elsworth, Y., Howe, R., Isaak, G. R., Larsen, R. M., New, R., Schou, J., Thompson, M. J. \& Tomczyk, S. 1999, MNRAS 308, 405

Charbonneau, P., Christensen-Dalsgaard, J., Henning, R., Larsen, R. M., Schou, J., Thompson, M. J. \& Tomczyk, S. 1999, ApJ 527, 445

Charbonnel, C. \& Talon, S. 2005, Science 309, 2189

Christensen-Dalsgaard, J. 2002, Rev. Mod. Phys. 74, 1073

Christensen-Dalsgaard, J. \& Schou, J. 1988, in: V. Domingo \& E. J. Rolfe (eds), Seismology of the Sun \& Sun-like Stars, ESA SP-286, p. 149

Christensen-Dalsgaard, J. \& Thompson, M. J. 2007, in: D. W. Hughes, R. Rosner \& N. O. Weiss, (eds), The solar tachocline (Cambridge University Press), in the press

Christensen-Dalsgaard, J., Gough, D. O. \& Thompson, M. J. 1991, ApJ 378, 413

Corbard, T. \& Thompson, M. J. 2002, Solar Phys. 205, 211

Corbard, T., Blanc-Féraud, L., Berthomieu, G. \& Provost, J. 1999, A\&GA 344, 696

Covas, E., Tavakol, R., Moss, D. \& Tworkowski, A. 2000, A\&SA 360, L21

DeRosa, M. L., Gilman, P. A. \& Toomre, J. 2002, ApJ 581, 1356

Deubner, F.-L., Ulrich, R. K. \& Rhodes, E. J. 1979, A\& $A$ 72, 177

Duvall, T. L., Dziembowski, W. A., Goode, P. R., Gough, D. O., Harvey, J. W. \& Leibacher, J. W. 1984, Nature 310, 22

Dziembowski, W. A., Goode, P. R. \& Libbrecht, K. G. 1989, ApJ 337, L53

Eggenberger, P., Maeder, A. \& Meynet, G. 2005, A\& $A$ 440, L9

Foukal, P. 1972, ApJ 173, 439

García, R. A., Turck-Chièze, S., Jiménez-Reyes, S. J., Ballot, J., Pallé, P., Eff-Darwich, A., Mathur, S. \& Provost, J. 2006, in: K. Fletcher (ed.), Proc. SOHO 18/GONG 2006/HELAS I Conf. Beyond the spherical Sun, ESA SP-624, in the press

Giles, P. M., Duvall Jr, T. L., Scherrer, P. H. \& Bogart, R. S. 1997, Nature 390, 52

Gilman, P. A. \& Howe, R. 2003, in: A. Wilson (ed.), Proc. SOHO 12/GONG+ 2002. Local and Global Helioseismology: The Present and Future, ESA SP-517, p. 283

Gilman, P. A. \& Miller, J. 1986, ApJS 61, 585

Gizon, L. \& Birch, A. C. 2005, Living Rev. Solar Phys. 2, 6. URL (cited on 14/10/06): http://www.livingreviews.org/lrsp-2005-6

Glatzmaier, G. 1985, ApJ 291, 300

Gough, D. O. 1993, in: Zahn, J.-P. \& Zinn-Justin, J. (eds), Astrophysical fluid dynamics, Les Houches Session XLVII, (Elsevier, Amsterdam), p. 399

Gough, D. O. \& McIntyre, M. E. 1998, Nature 394, 755 
Gough, D. O. \& Toomre, J. 1983, Solar Phys. 82, 401

Haber, D. A., Hindman, B. W., Toomre, J., Bogart, R. S., Larsen, R. M. \& Hill, F. 2002, ApJ 570,855

Harvey, J. W., Hill, F., Hubbard, R. P., et al. 1996, Science 272, 1284

Hathaway, D. H. 1996, ApJ 460, 1027

Hill, F. 1988, ApJ 333, 996

Howard, R. \& LaBonte, B. J. 1980, ApJ 239, L33

Howe, R. 2006, in: K. Fletcher (ed.), Proc. SOHO 18/GONG 2006/HELAS I Conf. Beyond the spherical Sun, ESA SP-624, in the press

Howe, R., Christensen-Dalsgaard, J., Hill, F., Komm, R. W., Larsen, R. M., Schou, J., Thompson, M. J. \& Toomre, J., 2000a, Science 287, 2456

Howe, R., Christensen-Dalsgaard, J., Hill, F., Komm, R. W., Larsen, R. M., Schou, J., Thompson, M. J. \& Toomre, J., 2000b, ApJ 533, L163

Howe, R., Komm, R., Hill, F., Ulrich, R., Haber, D. A., Hindman, B. W., Schou, J. \& Thompson, M. J., 2006a, Solar Phys. 235, 1

Howe, R., Rempel, M., Christensen-Dalsgaard, J., Hill, F., Komm, R., Larsen, R. M., Schou, J. \& Thompson, M. J., 2006b, ApJ 649, 1155

Howe, R., Christensen-Dalsgaard, J., Hill, F., Komm, R., Schou, J., Thompson, M. J. \& Toomre, J. 2007, in: G. Maris, K. Mursula \& I. Usoskin (eds), Proc. of the Second International Symposium on Space Climate, Adv. Space Res., submitted

Kosovichev, A. G. 1996, ApJ 469, L61

Kosovichev, A. G. \& Schou, J. 1997, ApJ 482, L207

Miesch, M. S. 2005, Living Rev. Solar Phys. 2, 1. URL (cited on 14/10/06): http://www . livingreviews.org/lrsp-2005-1

Miesch, M. S., Brun, A. S. \& Toomre, J. 2006, ApJ 641, 618

Pedlosky, J. 1987, Geophysical Fluid Dynamics, 2nd Ed., Springer-Verlag, New York.

Rempel, M. 2005, ApJ 622, 1320

Rempel, M., 2006a, ApJ 647, 662

Rempel, M., 2006b, ApJ, in the press [astro-ph/0610221 v1]

Scherrer, P. H., Bogart, R. S., Bush, R. I., et al. 1995, Solar Phys. 162, 129

Schou, J. 1999, ApJ 523, L181

Schou, J., Antia, H. M., Basu, S., et al. 1998, ApJ 505, 390

Schou, J., Howe, R., Basu, S., Christensen-Dalsgaard, J., Corbard, T., Hill, F., Larsen, R. M., Rabello-Soares, M. C. \& Thompson, M. J. 2002, ApJ 567, 1234

Spiegel, E. A. \& Zahn, J.-P. 1992, A\&SA 265, 106

Spruit, H. C. 2003, Solar Phys. 213, 1

Thompson, M. J., Christensen-Dalsgaard, J., Miesch, M. S. \& Toomre, J. 2003, ARAA 41, 599

Toomre, J., Christensen-Dalsgaard, J., Hill, F., Howe, R., Komm, R. W., Schou, J. \& Thompson, M. J. 2003, in: A. Wilson (ed.), Proc. SOHO 12 / GONG+ 2002. Local and Global Helioseismology: The Present and Future, ESA SP-517, p. 409

Unno, W., Osaki, Y., Ando, H., Saio, H. \& Shibahashi, H. 1989, Nonradial Oscillations of Stars, 2nd Edition (University of Tokyo Press)

Vorontsov, S. V., Christensen-Dalsgaard, J., Schou, J., Strakhov, V. N. \& Thompson, M. J. 2002, Science 296, 101

Wilcox, J. M. \& Howard, R. 1970, Solar Phys. 13, 251

Zhao, J. \& Kosovichev, A. G. 2004, ApJ 603, 776

\section{Discussion}

PECKER: The trend towards the equator seems to be much quicker (in your diagram) for torsional oscillations than it is for helioseismological data. Why is that so?

Christensen-Dalsgaard: In fact, my impression from Figure 7 is that the helioseismically inferred flow is quite similar to the behaviour of the surface torsional oscillation and, if anything, is at slightly lower latitude. This could be related to the apparent propagation towards the surface, as illustrated in Figure 8. 